\title{
Enhanced electroweak radiative corrections in SUSY and precision data
}

\author{
I.V. Gaidaenko, A.V. Novikov, V.A. Novikov \\ ITEP, Moscow, Russia \\ A.N. Rozanov \\ CPPM, IN2P3, CNRS, France and ITEP, Moscow, Russia \\ M.I. Vysotsky \\ ITEP, Moscow, Russia and INFN, Sezione di Ferrara, Italy \\ Submitted to Physics Reports
}

\begin{abstract}
Enhanced radiative corrections generated in SUSY extensions of the Standard Model spoil the fit of the precision data (Z-boson decay parameters and W-boson mass). This negative effect is washed out for heavy enough squarks, because of the decoupling property of SUSY models. We find that even for light squarks the enhanced radiative corrections can be small. In this case substantial $\tilde{\mathrm{t}}_{\mathrm{L}} \tilde{\mathrm{t}}_{\mathrm{R}}$ mixing is necessary.
\end{abstract}


A number of predictions of the Standard Model are now tested with an accuracy of the order of $0.1 \%$. There are two (more or less) free parameters in the Standard Model: the value of the strong coupling constant $\hat{\alpha}_{\mathrm{s}}\left(m_{\mathrm{Z}}\right)$ and the mass of the Higgs boson $m_{\mathrm{H}}$. Making a fit of the latest set of precision data reported in [1] (Z-boson decay parameters, W-boson and top-quark masses), we obtain:

$$
\begin{gathered}
m_{\mathrm{H}}=\left(71_{-43}^{+82}\right) \mathrm{GeV}, \\
\hat{\alpha}_{\mathrm{s}}\left(m_{\mathrm{Z}}\right)=0.119 \pm 0.003, \\
\chi^{2} / n_{\text {d.o.f. }}=15.0 / 14 .
\end{gathered}
$$

The quality of this fit is very good, which imposes strict constraints on the possible extensions of the Standard Model.

In the SUSY extensions a lot of new particles are introduced. Their contributions to electroweak observables come through loops and for $m_{\mathrm{SUSY}}>m_{\mathrm{W}}$ are of the order of $\alpha_{\mathrm{W}}\left(m_{\mathrm{W}} / m_{\mathrm{SUSY}}\right)^{2}$, where $m_{\mathrm{SUSY}}$ characterises the mass scale of the new particles. These contributions were calculated and analysed in number of papers [2]-[5].

A large violation of $\mathrm{SU}(2)$ symmetry in the third family of squarks by a large value of $m_{\mathrm{t}} \approx 175 \mathrm{GeV}$ leads to an enhancement of the corresponding oblique corrections by the factor $\left(m_{\mathrm{t}} / m_{\mathrm{W}}\right)^{4} \approx 16$ to be compared with the numerous terms of the order of $\alpha_{\mathrm{W}}\left(m_{\mathrm{W}} / m_{\mathrm{SUSY}}\right)^{2}$. (The presence of terms $\sim m_{\mathrm{t}}^{4}$ in SUSY models was recognised long ago [6].) Inspired by this fact, calculations of the enhanced corrections to the functions $V_{\mathrm{A}}, V_{\mathrm{R}}$ and $V_{\mathrm{m}}$ were made [7]. The functions $V_{i}$ describe electroweak radiative corrections to Z-boson couplings to leptons $g_{\mathrm{A}}$ and $g_{\mathrm{V}} / g_{\mathrm{A}}$, and to W-boson mass [8]. To calculate these enhanced terms we expanded the polarisation operators of the vector bosons $\Sigma_{\mathrm{V}}\left(k^{2}\right)$ at $k^{2}=0$. The terms enhanced as $m_{\mathrm{t}}^{4} / m_{\mathrm{W}}^{2} m_{\mathrm{SUSY}}^{2}$ come from $\Sigma_{\mathrm{W}}(0)$, while those enhanced as $m_{\mathrm{t}}^{2} / m_{\mathrm{SUSY}}^{2}$ come from $\Sigma_{\mathrm{W}, \mathrm{Z}}^{\prime}(0)$. The higher-order derivatives of self-energies are suppressed as $\left(m_{\mathrm{W}, \mathrm{Z}} / m_{\mathrm{SUSY}}\right)^{2}$, and are therefore not taken into account.

In the present paper the influence of these new terms on the precision data fit will be analysed. To begin with, we should expand the analysis of [7] and take into account the main SUSY corrections to hadronic Z-decays as well. Vertices with gluino exchange generate (potentially) large corrections of the order of $\hat{\alpha}_{\mathrm{s}}\left(m_{\mathrm{Z}} / m_{\mathrm{SUSY}}\right)^{2}$ in the limit $m_{\mathrm{SUSY}}>m_{\mathrm{Z}}$. For hadronic $\mathrm{Z}$ decays we use the following expression for the width [9]:

$$
\Gamma_{\mathrm{q}}=\Gamma_{\mathrm{Z} \rightarrow \mathrm{q} \overline{\mathrm{q}}}=12\left[g_{\mathrm{A}_{\mathrm{q}}}^{2} R_{\mathrm{Aq}_{\mathrm{q}}}+g_{\mathrm{V}_{\mathrm{q}}}^{2} R_{\mathrm{V}_{\mathrm{q}}}\right] \Gamma_{0},
$$


where $\Gamma_{0}=\frac{1}{24 \sqrt{2} \pi} G_{\mu} m_{\mathrm{Z}}^{3}$.

Corrections induced by gluino exchanges lead to the following SUSY shifts of the factors $R_{i_{\mathrm{q}}}$ [10:

$$
\begin{gathered}
\delta R_{\mathrm{V}_{\mathrm{q}}}=\delta R_{\mathrm{A}_{\mathrm{q}}}=1+\frac{\hat{\alpha}_{\mathrm{s}}\left(m_{\mathrm{Z}}\right)}{\pi} \Delta_{1}, \\
\Delta_{1}=-\frac{4}{3} \int_{0}^{1} d z_{1} \int_{0}^{1-z_{1}} d z_{2} \ln \left[1-\frac{x y z_{1} z_{2}}{x+\left(z_{1}+z_{2}\right)(y-x)}\right],
\end{gathered}
$$

where $x=\left(m_{\mathrm{Z}} / m_{\tilde{\mathrm{q}}}\right)^{2}, y=\left(m_{\mathrm{Z}} / m_{\tilde{\mathrm{g}}}\right)^{2}$. We take these strong SUSY corrections into account in our analysis. The weak SUSY corrections to $\mathrm{Z}$ decays into hadrons are taken into account by the corrections to the functions $V_{\mathrm{A}}$ and $V_{\mathrm{R}}$ calculated in [7].

The stop exchange contributes to the vertex corrections to the $\mathrm{Z} \rightarrow \mathrm{b} \overline{\mathrm{b}}$ decay amplitude; but since there are no terms enhanced as $\left(m_{\mathrm{t}} / m_{\mathrm{W}}\right)^{4}$,11], we will not take the corresponding corrections into account in the present paper.

Let us start the discussion of the SUSY corrections to the functions $V_{i}$ from the description of the stop sector of the theory. The $\tilde{t}_{L} \tilde{t}_{R}$ mass matrix has the following form:

$$
\left(\begin{array}{cc}
m_{\tilde{\mathrm{t}}_{\mathrm{L}}}^{2} & m_{\mathrm{t}} A_{\mathrm{t}}^{\prime} \\
m_{\mathrm{t}} A_{\mathrm{t}}^{\prime} & m_{\tilde{\mathrm{t}}_{\mathrm{R}}}^{2}
\end{array}\right)
$$

where $\tilde{t}_{L} \tilde{t}_{R}$ mixing is proportional to a large value of the top-quark mass and is not small. Diagonalizing matrix (]) we obtain the following eigenstates:

$$
\left\{\begin{array}{l}
\tilde{t}_{1}=c_{\mathrm{u}} \tilde{\mathrm{t}}_{\mathrm{L}}+s_{\mathrm{u}} \tilde{\mathrm{t}}_{\mathrm{R}} \\
\tilde{t}_{2}=-s_{\mathrm{u}} \tilde{\mathrm{t}}_{\mathrm{L}}+c_{\mathrm{u}} \tilde{\mathrm{t}}_{\mathrm{R}}
\end{array}\right.
$$

where $c_{\mathrm{u}} \equiv \cos \theta_{\mathrm{LR}}, s_{\mathrm{u}} \equiv \sin \theta_{\mathrm{LR}}, \theta_{\mathrm{LR}}$ being $\tilde{t}_{L} \tilde{t}_{R}$ mixing angle, and

$$
\tan ^{2} \theta_{\mathrm{LR}}=\frac{m_{1}^{2}-m_{\tilde{\mathrm{t}}_{\mathrm{L}}}^{2}}{m_{\tilde{\mathrm{t}}_{\mathrm{L}}}^{2}-m_{2}^{2}}, \quad m_{1}^{2} \geq m_{\tilde{\mathrm{t}}_{\mathrm{L}}}^{2} \geq m_{2}^{2},
$$

where $m_{1}$ and $m_{2}$ are the mass eigenvalues:

$$
m_{1,2}^{2}=\frac{m_{\tilde{\mathrm{t}}_{\mathrm{L}}}^{2}+m_{\tilde{\mathrm{t}}_{\mathrm{R}}}^{2}}{2} \pm \frac{\left|m_{\tilde{\mathrm{t}}_{\mathrm{L}}}^{2}-m_{\tilde{\mathrm{t}}_{\mathrm{R}}}^{2}\right|}{2} \sqrt{1+\frac{4 m_{\mathrm{t}}^{2} A_{\mathrm{t}}^{2}}{\left(m_{\tilde{\mathrm{t}}_{\mathrm{L}}}^{2}-m_{\tilde{\mathrm{t}}_{\mathrm{R}}}^{2}\right)^{2}}} .
$$


The following relation between $m_{\tilde{\mathrm{t}}_{\mathrm{L}}}^{2}$ and $m_{\tilde{\mathrm{b}}_{\mathrm{L}}}^{2}$ takes place:

$$
m_{\tilde{\mathrm{t}}_{\mathrm{L}}}^{2}=m_{\tilde{\mathrm{b}}_{\mathrm{L}}}^{2}+m_{\mathrm{t}}^{2}+m_{\mathrm{Z}}^{2} \cos (2 \beta) c_{W}^{2}
$$

where $c_{\mathrm{W}}^{2} \equiv \cos ^{2} \theta_{\mathrm{W}}=0.77\left(\theta_{\mathrm{W}}\right.$ is the electroweak mixing angle) and $\tan \beta$ is equal to the ratio of the vacuum averages of two Higgs neutrals, introduced in SUSY models. Relation (11) is central for the present paper; it demonstrates a large breaking of $\mathrm{SU}(2)$ symmetry in the third generation of squarks. The only hypothesis that is behind this relation is that the main origin of the large breaking of this $\mathrm{SU}(2)$ is in the quark Higgs interaction.

The enhanced electroweak radiative corrections induced by squarks of the third generation depend on 3 parameters: $m_{1}, m_{2}$ and $m_{\tilde{\mathrm{b}}_{\mathrm{L}}}$. The dependence on the angle $\beta$ is very moderate and, in numerical fits, we will use the rather popular value of $\tan \beta=2$. In what follows we will write $m_{\tilde{\mathrm{b}}}$ instead of $m_{\tilde{\mathrm{b}}_{\mathrm{L}}}$, bearing in mind that the $\tilde{b}_{\mathrm{R}}$ squark does not contribute to the corrections under investigation (let us note that $\tilde{\mathrm{b}}_{\mathrm{L}} \tilde{\mathrm{b}}_{\mathrm{R}}$ mixing is proportional to $m_{\tilde{\mathrm{b}}}$ and can be neglected).

Let us present the formulas from [7], which describe the enhanced SUSY corrections to the functions $V_{i}$ :

$$
\begin{aligned}
& \delta_{\mathrm{SUSY}}^{\mathrm{LR}} V_{\mathrm{A}}=\frac{1}{m_{\mathrm{Z}}^{2}}\left[c_{\mathrm{u}}^{2} g\left(m_{1}, m_{\tilde{\mathrm{b}}}\right)+s_{\mathrm{u}}^{2} g\left(m_{2}, m_{\tilde{\mathrm{b}}}\right)-c_{\mathrm{u}}^{2} s_{\mathrm{u}}^{2} g\left(m_{1}, m_{2}\right)\right] \\
& \delta_{\mathrm{SUSY}}^{\mathrm{LR}} V_{\mathrm{R}}=\delta_{\mathrm{SUSY}}^{\mathrm{LR}} V_{\mathrm{A}}+\frac{1}{3} Y_{\mathrm{L}}\left[c_{\mathrm{u}}^{2} \ln \left(\frac{m_{1}^{2}}{m_{\tilde{\mathrm{b}}}^{2}}\right)+s_{\mathrm{u}}^{2} \ln \left(\frac{m_{2}^{2}}{m_{\tilde{\mathrm{b}}}^{2}}\right)\right]-\frac{1}{3} c_{\mathrm{u}}^{2} s_{\mathrm{u}}^{2} h\left(m_{1}, m_{2}\right) \\
& \delta_{\mathrm{SUSY}}^{\mathrm{LR}} V_{\mathrm{m}}=\delta_{\mathrm{SUSY}}^{\mathrm{LR}} V_{\mathrm{A}}+\frac{2}{3} Y_{\mathrm{L}} s^{2}\left[c_{\mathrm{u}}^{2} \ln \left(\frac{m_{1}^{2}}{m_{\tilde{\mathrm{b}}}^{2}}\right)+s_{\mathrm{u}}^{2} \ln \left(\frac{m_{2}^{2}}{m_{\tilde{\mathrm{b}}}^{2}}\right)\right]+ \\
&+\frac{c^{2}-s^{2}}{3}\left[c_{\mathrm{u}}^{2} h\left(m_{1}, m_{\tilde{\mathrm{b}}}\right)+s_{\mathrm{u}}^{2} h\left(m_{2}, m_{\tilde{\mathrm{b}}}\right)\right]-\frac{c_{\mathrm{u}}^{2} s_{\mathrm{u}}^{2}}{3} h\left(m_{1}, m_{2}\right)
\end{aligned}
$$

where

$$
g\left(m_{1}, m_{2}\right)=m_{1}^{2}+m_{2}^{2}-2 \frac{m_{1}^{2} m_{2}^{2}}{m_{1}^{2}-m_{2}^{2}} \ln \left(\frac{m_{1}^{2}}{m_{2}^{2}}\right)
$$




$$
h\left(m_{1}, m_{2}\right)=-\frac{5}{3}+\frac{4 m_{1}^{2} m_{2}^{2}}{\left(m_{1}^{2}-m_{2}^{2}\right)^{2}}+\frac{\left(m_{1}^{2}+m_{2}^{2}\right)\left(m_{1}^{4}-4 m_{1}^{2} m_{2}^{2}+m_{2}^{4}\right)}{\left(m_{1}^{2}-m_{2}^{2}\right)^{3}} \ln \left(\frac{m_{1}^{2}}{m_{2}^{2}}\right),
$$

and $Y_{\mathrm{L}}$ is the left-doublet hypercharge, $Y_{\mathrm{L}}=Q_{\mathrm{t}}+Q_{\mathrm{b}}=1 / 3$.

Now that we have all the necessary formulas at our disposal, let us start with analysing the data in the simplest case of the absence of $\tilde{t}_{\mathrm{L}} \tilde{t}_{R}$ mixing, $\sin \theta_{\mathrm{LR}}=0$. In this case the corrections $\delta_{\mathrm{SUSY}} V_{i}$ depend on parameter $m_{\tilde{\mathrm{b}}}$ only, and this dependence is shown in Figs. $1-3$ of paper [7]. For $m_{\tilde{b}} \lesssim$ $300 \mathrm{GeV}$ the theoretical values of all 3 functions $V_{i}$ become larger than the experimental values. Let us begin our fit by taking the value of the Higgs boson mass as a free parameter. In this fit, the mass of the Higgs boson

\begin{tabular}{|c|c|c|c|}
\hline$m_{\tilde{\mathrm{b}}}(\mathrm{GeV})$ & $m_{\mathrm{H}}(\mathrm{GeV})$ & $\hat{\alpha}_{\mathrm{s}}$ & $\chi^{2} / n_{\text {d.o.f. }}$ \\
\hline 100 & $850_{-320}^{+286}$ & $0.113 \pm 0.003$ & $20.3 / 14$ \\
150 & $484_{-235}^{+364}$ & $0.116 \pm 0.003$ & $18.1 / 14$ \\
200 & $280_{-144}^{+240}$ & $0.117 \pm 0.003$ & $17.3 / 14$ \\
300 & $152_{-87}^{+145}$ & $0.118 \pm 0.003$ & $16.3 / 14$ \\
400 & $113_{-68}^{+115}$ & $0.119 \pm 0.003$ & $15.8 / 14$ \\
1000 & $77_{-47}^{+87}$ & $0.119 \pm 0.003$ & $15.2 / 14$ \\
\hline
\end{tabular}

Table 1: Fit of the precision data with SUSY corrections taken into account in the case of the absence of $\tilde{\mathrm{t}}_{\mathrm{L}} \tilde{\mathrm{t}}_{\mathrm{R}}$ mixing, $\sin \theta_{\mathrm{LR}}=0$ and $m_{\mathrm{H}}$ taken as a free parameter. For $m_{\tilde{\mathrm{b}}}>300 \mathrm{GeV}$, SUSY corrections become negligible and the SM fit of the data is reproduced.

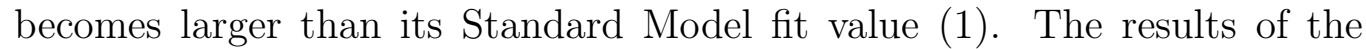
fit are shown in Table \&. (To reduce the number of parameters we take $m_{\tilde{\mathrm{g}}}=m_{\tilde{\mathrm{b}}}$ in this paper. Let us stress that light quarkinos with masses of the order of $100-200 \mathrm{GeV}$ are usually allowed only if gluinos are heavy, 
$m_{\tilde{\mathrm{g}}} \geq 500 \mathrm{GeV}$ [12]. In the case of the heavy gluino correction $\Delta_{1}$ (eq. (66)) becomes power-suppressed and we return to the Standard Model fit value of $\hat{\alpha}_{\mathrm{s}}=0.119$.) We see how in the SUSY extension of the Standard Model with light superpartners the fit gets worse. However, in SUSY the mass of the Higgs boson is no longer a free parameter. Of the 3 neutral Higgs bosons, the lightest should have a mass less than approximately $120 \mathrm{GeV}$. If other Higgs bosons are considerably heavier, this lightest boson has the same couplings to gauge bosons as in the Standard Model, so formulas for the Standard Model radiative corrections can be used since deviations are suppressed as $\left(m_{\mathrm{h}} / m_{\mathrm{A}}\right)^{2}\left(m_{\mathrm{A}}\right.$ being the mass of the heaviest Higgs). For the

\begin{tabular}{|c|c|c|}
\hline$m_{\tilde{b}}(\mathrm{GeV})$ & $\hat{\alpha}_{\mathrm{s}}$ & $\chi^{2} / n_{\text {d.o.f. }}$ \\
\hline 100 & $0.110 \pm 0.003$ & $30.2 / 15$ \\
150 & $0.115 \pm 0.003$ & $21.9 / 15$ \\
200 & $0.116 \pm 0.003$ & $18.6 / 15$ \\
300 & $0.118 \pm 0.003$ & $16.4 / 15$ \\
400 & $0.119 \pm 0.003$ & $15.8 / 15$ \\
1000 & $0.119 \pm 0.003$ & $15.5 / 15$ \\
\hline
\end{tabular}

Table 2: The same as Table 1, but with a value of the lightest Higgs-boson mass $m_{\mathrm{h}}=120 \mathrm{GeV}$ that is about the maximum allowed value in the simplest SUSY models.

maximal allowed value $m_{\mathrm{h}}=120 \mathrm{GeV}$, the results of the fit are shown in Table 2. In what follows, we will always take $m_{\mathrm{h}}=120 \mathrm{GeV}$ since, for 90 $\mathrm{GeV}<m_{\mathrm{h}}<120 \mathrm{GeV}$, the results of the fit are practically the same. This table demonstrates that superpartners should be heavy if we want to have a good-quality fit of the data. The next step is to take into account $\tilde{t}_{L} \tilde{t}_{R}$ mixing.

In Fig. 11 we show the dependence of SUSY corrections $\delta_{\mathrm{SUSY}} V_{i}$ on $m_{1}$ and $m_{2}$ for $m_{\tilde{\mathrm{b}}}=150 \mathrm{GeV}$; the same in Fig. 目 for $m_{\tilde{\mathrm{b}}}=200 \mathrm{GeV}$. One clearly sees from these figures that there exists, even for such low values of $m_{\tilde{\mathrm{b}}}$, domain of low $m_{2}$ values where the enhanced radiative corrections are damped. In Fig.2 there is a valley where $\delta_{\mathrm{SUSY}} V_{i}$ reach the minimum values, which are considerably smaller than 1 . This valley starts at $m_{2} \approx m_{\tilde{\mathrm{b}}}, m_{1} \approx 1000$ $\mathrm{GeV}$ and goes to $m_{2} \approx 100 \mathrm{GeV}, m_{1} \approx 400 \mathrm{GeV}$. The smallness of the radiative corrections at the point $m_{2} \approx m_{\tilde{\mathrm{b}}}, m_{1} \approx 1000 \mathrm{GeV}$ can be easily understood: here $\theta_{\mathrm{LR}} \approx \pi / 2$, so in $\delta_{\mathrm{SUSY}} V_{A}$ only the term proportional to 
$g\left(m_{2}, m_{\tilde{\mathrm{b}}}\right)$ remains. However, for $m_{2}=m_{\tilde{\mathrm{b}}}$ this term equals zero. At this end-point of the valley, $\tilde{\mathrm{t}}_{2} \approx \tilde{\mathrm{t}}_{\mathrm{L}}, \tilde{\mathrm{t}}_{1} \approx \tilde{\mathrm{t}}_{\mathrm{R}}$, so $m_{\tilde{\mathrm{t}}_{\mathrm{R}}}^{2} \gg m_{\tilde{\mathrm{t}}_{\mathrm{L}}}^{2}$, which prevents the relation between $m_{\tilde{\mathfrak{t}}_{\mathrm{R}}}$ and $m_{\tilde{\mathrm{t}}_{\mathrm{L}}}$ occurring in a wide class of models. In these models (e.g. in the MSSM) the left and the right squark masses are equal at the high energy scale and, renormalizing them to low energies we have $m_{\tilde{\mathrm{t}}_{\mathrm{L}}}^{2}>m_{\tilde{\mathrm{t}}_{\mathrm{R}}}^{2}$. Almost along the whole valley we have $\tan ^{2} \theta_{\mathrm{LR}}>1$, which means that $m_{\tilde{\mathrm{t}}_{\mathrm{R}}}^{2}>m_{\tilde{\mathrm{t}}_{\mathrm{L}}}^{2}$. This possibility to suppress radiative corrections was discussed in [13]. However, in the vicinity of the end-point $m_{1}=300 \mathrm{GeV}$, $m_{2}=70 \mathrm{GeV}$ the value of $\tan ^{2} \theta_{\mathrm{LR}}$ becomes smaller than 1 and $m_{\tilde{\mathrm{t}}_{\mathrm{R}}}^{2}<m_{\tilde{\mathrm{t}}_{\mathrm{L}}}^{2}$.

\begin{tabular}{|c|c|c|}
\hline$m_{1}(\mathrm{GeV})$ & $\hat{\alpha}_{\mathrm{s}}$ & $\chi^{2} / n_{\text {d.o.f. }}$ \\
\hline 482 & $0.116(3)$ & $16.3 / 15$ \\
743 & $0.117(3)$ & $15.8 / 15$ \\
1289 & $0.117(3)$ & $15.6 / 15$ \\
\hline
\end{tabular}

Table 3: For light $m_{\tilde{\mathrm{b}}}=150 \mathrm{GeV}$, light $m_{2}=m_{\tilde{\mathrm{b}}}$ and $m_{\mathrm{h}}=120 \mathrm{GeV}$, the quality of the fit can be the same as in the Standard Model, if $m_{1}$ is heavy enough.

In Table 3 we present the results of the fit, assuming $m_{\tilde{\mathrm{b}}}=150 \mathrm{GeV}$ and $m_{\mathrm{h}}=120 \mathrm{GeV}$, along the line of minimum $\chi^{2}$, which is formed at $m_{2} \approx 150$ $\mathrm{GeV}$. We see that for heavy $m_{1}$ the quality of the fit is not worse than in the Standard Model. In Table 1 we show values of $\chi^{2}$ along its valley of

\begin{tabular}{|c|c|c|c|}
\hline$m_{1}(\mathrm{GeV})$ & $m_{2}(\mathrm{GeV})$ & $\hat{\alpha}_{\mathrm{s}}$ & $\chi^{2} / n_{\text {d.o.f. }}$ \\
\hline 1296 & 193 & $0.118 \pm 0.003$ & $15.6 / 15$ \\
888 & 167 & $0.118 \pm 0.003$ & $15.8 / 15$ \\
387 & 131 & $0.118 \pm 0.003$ & $16.1 / 15$ \\
296 & 72 & $0.117 \pm 0.003$ & $16.7 / 15$ \\
\hline
\end{tabular}

Table 4: For fixed values of $m_{\tilde{\mathrm{b}}}=200 \mathrm{GeV}$ and $m_{\mathrm{h}}=120 \mathrm{GeV}$, results of the fit along the valley of minimum $\chi^{2}$.

minimum, which is formed for $m_{\tilde{\mathrm{b}}}=200 \mathrm{GeV}$. Once more we observe that a good quality of the fit is possible for light superpartners if $\tilde{t}_{L} \tilde{t}_{R}$ mixing is taken into account. This effect is clearly seen as a valley of low $\chi^{2}$ on the 
plots of the allowed regions in the plane $m_{1}-m_{2}$ both for $m_{\tilde{\mathrm{b}}}=150 \mathrm{GeV}$ on Fig. 3-a and for $m_{\tilde{\mathrm{b}}}=200 \mathrm{GeV}$ on Fig. 3-b.

The formulas for the enhanced radiative corrections in SUSY extensions of the Standard Model obtained in [7] were used in the present paper to fit the data of the precision measurements of Z-boson decay parameters at LEP and the SLC, the value of $m_{\mathrm{W}}$ and $m_{\mathrm{t}}$ at the Tevatron. The fit with SUSY corrections, assuming a small value of $m_{\tilde{b}}$, the absence of $\tilde{\mathrm{t}}_{\mathrm{L}} \tilde{\mathrm{t}}_{\mathrm{R}}$ mixing, and $m_{\mathrm{h}}=120 \mathrm{GeV}$, leads to the growth of the $\chi^{2}$ value. Thanks to the decoupling property of the SUSY extension in the case of heavy squarks, the results of the Standard Model fit are reproduced. However, even for comparatively light sbottom and small mass of one of the two stops, values of $\tilde{\mathrm{t}}_{\mathrm{L}} \tilde{\mathrm{t}}_{\mathrm{R}}$ mixing can be found that have $\delta_{\mathrm{SusY}} V_{i}$ small and $\chi^{2}$ almost the same as in the Standard Model.

We are grateful to L.B. Okun for stimulating discussions. A.V. Novikov is grateful to G. Fiorentini for his hospitality in Ferrara, where part of this work was done. A.N. Rozanov is grateful to CPPM-IN2P3-CNRS for supporting this work. The research of I.G., A.N., V.N. and M.V. is supported by RFBR grants 96-02-18010, 96-15-96578, 98-02-17372 and 98-02-17453; that of A.N., V.N. and M.V. by INTAS-RFBR grant 95-05678 as well. We thanks Susy Vascotto for the kind help in the preparation of the manuscript. 


\section{References}

[1] LEP EWWG and SLD HFEWG, CERN-EP/99-15, 1999.

[2] P. Chankowski, A. Dabelstein, W. Hollik, W. Mösle, S. Pokorsky and J. Rosiek, Nucl. Phys. B417 (1994) 101.

[3] D. Garcia, R.J. Jimenez and J. Sola, Phys. Lett. B347 (1995) 309, 321.

[4] W. de Boer, A. Dabelstein, W. Hollik, W. Mösle and U. Schwickerath, Z. Phys. C75 (1997) 627.

[5] J. Erler and D.M. Pierce, Nucl. Phys. B526 (1998) 53.

[6] L. Alvarez-Gaumé, J. Polchinski and M. Wise, Nucl. Phys. B211 (1983) 495;

R. Barbieri and L. Maiani, Nucl. Phys. B224 (1983) 32.

[7] I.V. Gaidaenko, A.V. Novikov, V.A. Novikov, A.N. Rozanov and M.I. Vysotsky, JETP Lett. 67 (1998) 761.

[8] V.A. Novikov, L.B. Okun and M.I. Vysotsky, Nucl. Phys. B397 (1993) 35 ;

M.I. Vysotsky, V.A. Novikov, L.B. Okun and A.N. Rozanov, Usp. Fiz. Nauk 166 (1998) 539.

[9] V. Novikov, L. Okun, A. Rozanov and M. Vysotsky, LEPTOP, preprint ITEP 19-95/CPPM-95-1.

[10] K. Hagiwara and H. Murayama, Phys. Lett. 246B (1990) 533.

[11] M. Boulware and D. Finnell, Phys. Rev. D44 (1991) 2054.

[12] S. Lammel, FERMILAB-CONF-98-055-E (1998).

[13] P.Chankowski, Proc. Quantum effects in the MSSM, Barcelona 1997, p.87, preprint IFT/97-18, hep-ph/9711470. 

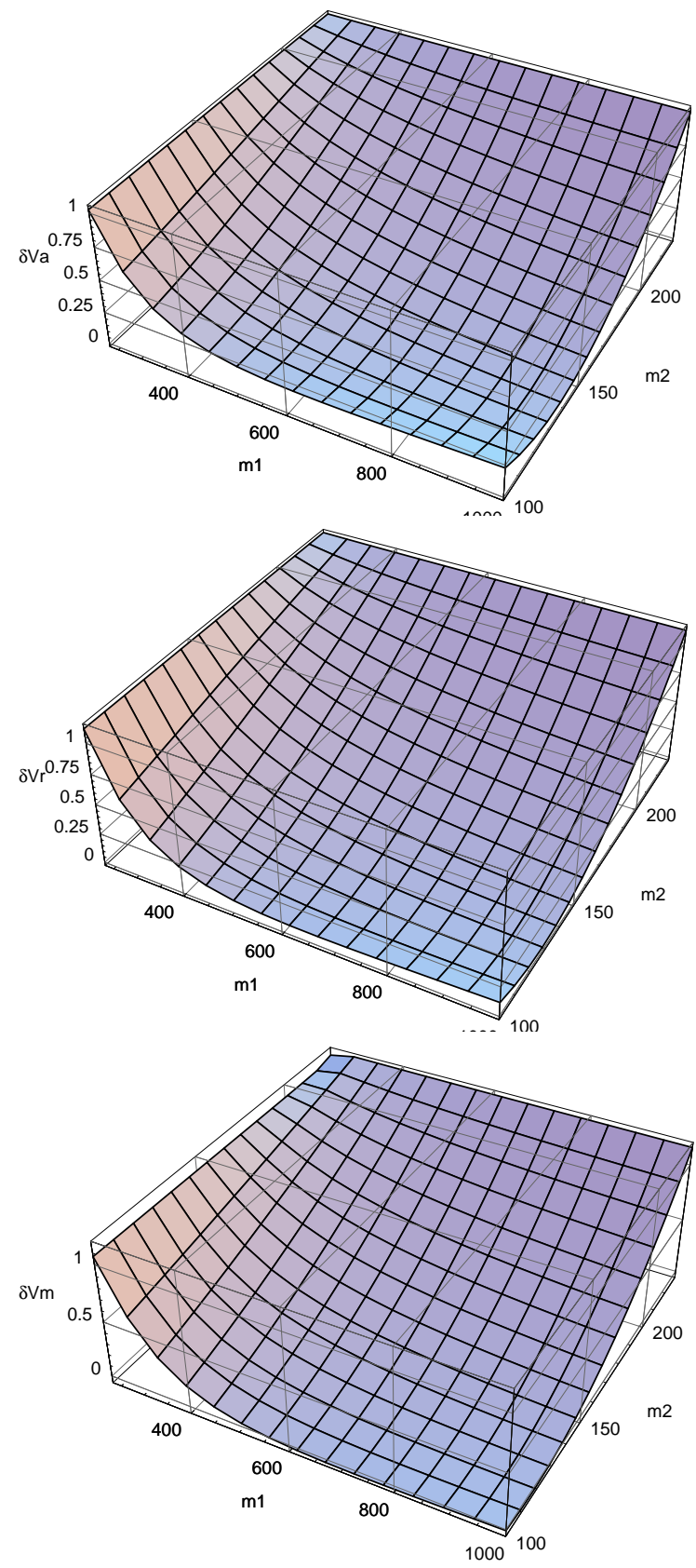

Figure 1: Values of the $\delta V_{\mathrm{A}}, \delta V_{\mathrm{R}}$ and $\delta V_{\mathrm{m}}$ at $m_{\tilde{\mathrm{b}}}=150 \mathrm{GeV}$ 

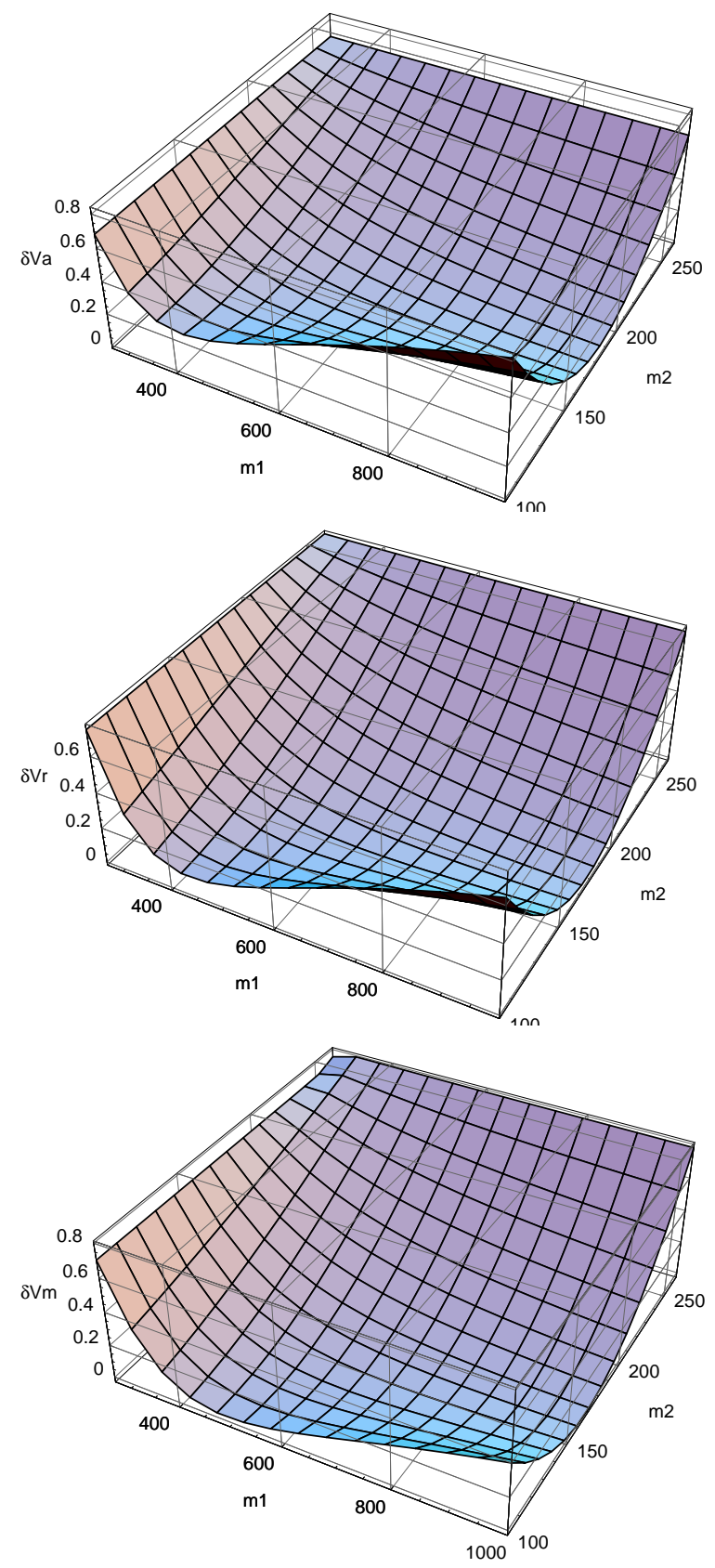

Figure 2: Values of the $\delta V_{\mathrm{A}}, \delta V_{\mathrm{R}}$ and $\delta V_{\mathrm{m}}$ at $m_{\tilde{\mathrm{b}}}=200 \mathrm{GeV}$ 


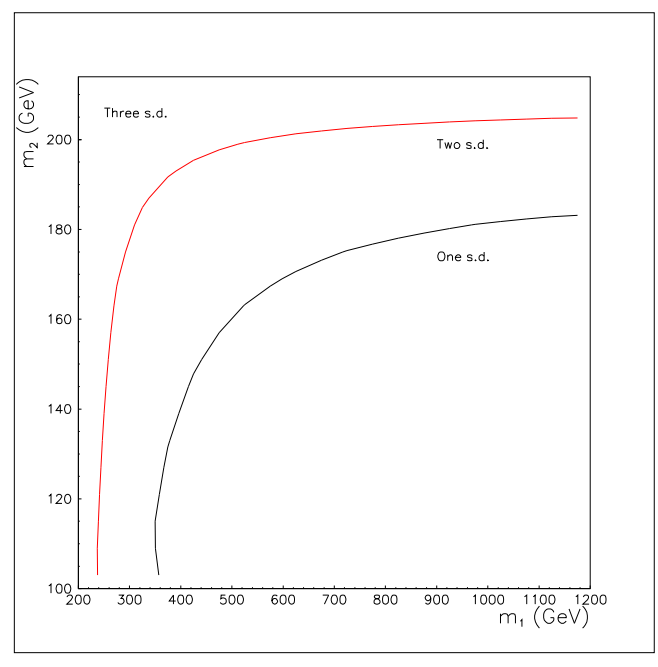

(a)

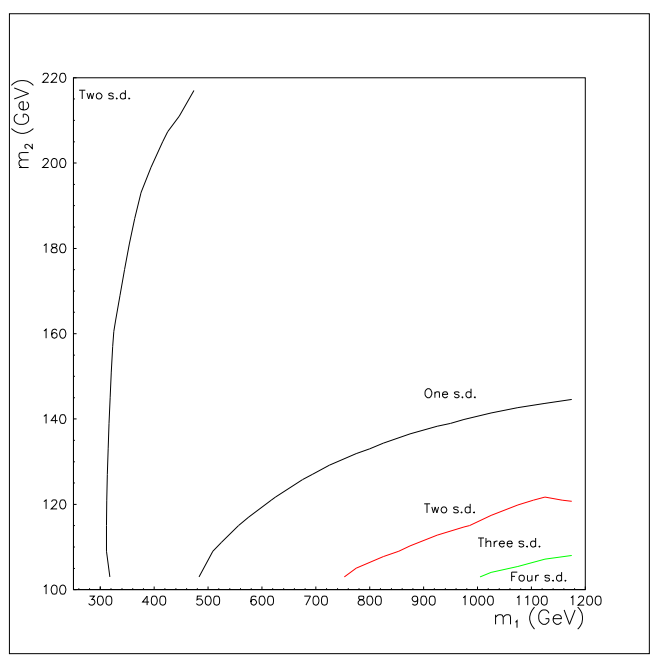

(b)

Figure 3: $m_{1}-m_{2}$ exclusion plot for SUSY model, assuming $m_{\mathrm{h}}=120 \mathrm{GeV}$, $m_{\tilde{\mathrm{g}}}=m_{\tilde{\mathrm{b}}}=m_{\tilde{\mathrm{b}}_{\mathrm{L}}}, \tan (\beta)=2$ and eq.(11). Three-parameter fit $\left(m_{\mathrm{t}}, \hat{\alpha}_{\mathrm{s}}, \bar{\alpha}\right)$ is done to EWWG-99 data. Figure (a) corresponds to $m_{\tilde{\mathrm{b}}}=150 \mathrm{GeV}$, while (b) corresponds to $m_{\tilde{\mathrm{b}}}=200 \mathrm{GeV}$. 